\title{
Psychological impact of COVID-19 on radiology: Emotional wellbeing versus psychological burnout
}

\author{
Abhishek Mahajan, Prerit Sharma' \\ Tata Memorial Hospital, Mumbai, 'Sharma Diagnostic Centre, Wardha, Maharashtra, India
}

Correspondence: Prof. Abhishek Mahajan, Department of Radiodiagnosis, Tata Memorial Hospital, Tata Memorial Centre, Mumbai, Maharashtra, India.E-mail: drabhishek.mahajan@yahoo.in

\begin{abstract}
While the COVID-19 pandemics seem to be crippling the global health care system, it has caused enormous uncertainty and panic in the lives of the health care professionals with a significant impact on their productivity. A significant proportion of these affected health care individuals comprise of the working staff belonging to the Radiology department. Although the main focus of all health care individuals has constantly been to reduce the number of new cases by minimal transmission, save lives of the infected patients by findings effective cure and carry out meticulous research to develop preventive vaccine, we have been unknowingly giving very little consideration to critical issues relating to the psychological well-being of the front liners. It is of utmost necessity that the psychological impact of this pandemic needs to be recognized and dealt with, in a timely fashion. While planning interventions to fight the pandemic, mental health issues must not be sidelined instead considered pragmatically. The radiology departments must come up with a mitigation plan to combat the subsequent short-term and long-term adverse effects of COVID-19 on mental well-being of their staff members. This article discusses the psychological impact of COVID-19 on the radiology department.
\end{abstract}

Key words: COVID-19; health care workers; psychological; radiographers; radiologist; residents; stress

\section{Introduction}

COVID-19 pandemic seems to be endlessly impacting the entire medical fraternity with its adverse effects on the physical as well as psychological state of the health care workers. Currently, we are all experiencing physical exhaustion and a significantly unstable emotional state of mind. Pandemics such as plague outbreaks have been known to mankind since times immemorial with Cholera and the Flu pandemics as the highlights of the nineteenth century and while COVID-19 is not the first pandemic that has been contracted by humanity, this pandemic is surely leaving deeply scarred memories and traumatic

\begin{tabular}{|l|l|}
\hline \multicolumn{2}{|c|}{ Access this article online } \\
\hline Quick Response Code: & \\
\hline & Website: \\
\hline
\end{tabular}

experiences for most people across the world. As per CNN, the pandemic impact on our civilization is going to be comparable to World War II and as COVID-19 continues to spread at a galloping pace, it has been ravaging one country after another. ${ }^{[1]}$

Since the beginning of the COVID-19 pandemic, radiology has played a significant role in the early diagnosis and effective management of the infected cases. This has resulted in an overburdened radiology department having led

This is an open access journal, and articles are distributed under the terms of the Creative Commons Attribution-NonCommercial-ShareAlike 4.0 License, which allows others to remix, tweak, and build upon the work non-commercially, as long as appropriate credit is given and the new creations are licensed under the identical terms.

For reprints contact: WKHLRPMedknow_reprints@wolterskluwer.com

Cite this article as: Mahajan A, Sharma P. Psychological impact of COVID-19 on radiology: Emotional wellbeing versus psychological burnout. Indian J Radiol Imaging 2021;31:S11-4.

Received: $10-\mathrm{Jul}-2020$ Accepted: $12-\mathrm{Sep}-2020$ Published: 23-Jan-2021 
to major psychological distress and trauma amongst the various cadre of working staff. ${ }^{[2,3]}$ The various factors that have contributed to these troubling circumstances include, social distancing, nation-wide lockdown, fear of one-self or loved ones contracting the disease, fear of isolation/ quarantine, shortage of adequate personal protective equipment and lack of guidance while deployed on clinical duties outside the radiology department [Figure 1]. The psychological impact includes acute stress disorder, posttraumatic stress disorder, and burnout. ${ }^{[4,5]}$ Let us start with brushing up our knowledge of definitions pertaining to these distressing mental conditions. Acute stress disorder is defined as an intense, unpleasant, and dysfunctional reaction that begins soon after an overwhelming traumatic episode and lasting less than a month. If these symptoms persist beyond a month, the individual is diagnosed to have posttraumatic stress disorder (PTSD).Similarly, burnout is a syndrome that is caused by increased exposure to stressors at the workplace that leads to emotional exhaustion, depersonalization, and diminished sense of personal achievement. ${ }^{[6]}$ Both individual and system based interventions can help cope up with the psychological impact of covid-19 [Figure 2].

Looking at the present scenario, India is presently demonstrating a community spread of COVID-19 and there is very little certainty of how this pandemic is going to unfold here further. A large part of the psychological responses has so far been reactionary to what has happened in other countries in the world, fears of what might be in store in the times ahead and responses to the lockdown. Here we discuss the psychological impact of COVID-19 on the radiology department on both the front liner radiographers, and the radiologists serving as the backbone.

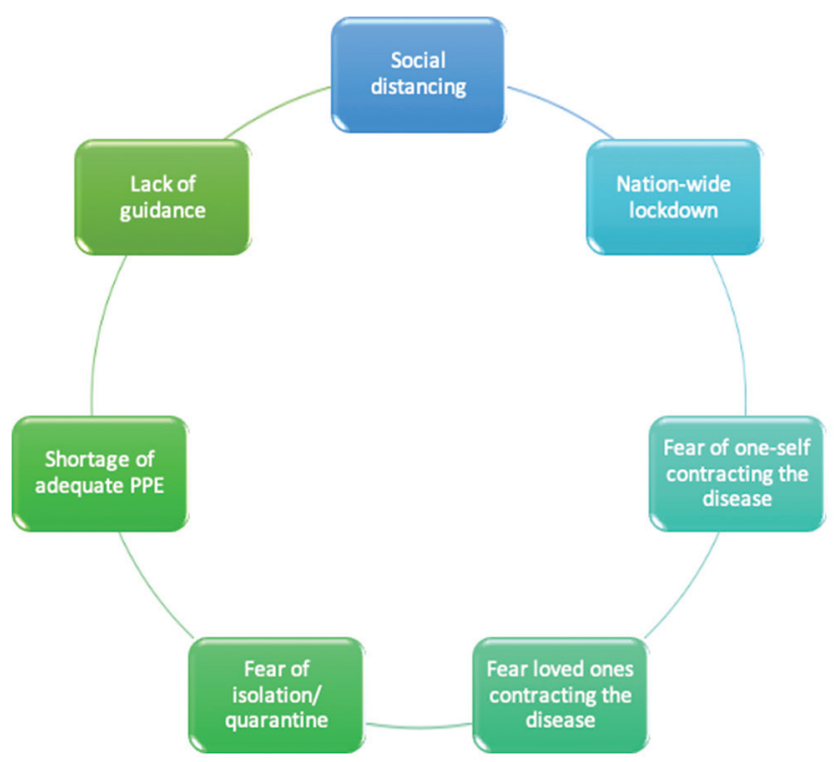

Figure 1: Factors influencing morale during the COVID-19 pandemic
Psychological issues and mitigation plan relating to the radiologists

Radiology faculty who have been actively involved in the management of the COVID-19 comprise of the residents, the junior and the senior consultant radiologists. In 2003, it was estimated that approximately $50 \%$ of healthcare workers had suffered from PTSD that was caused due to the severe acute respiratory syndrome (SARS) outbreak. ${ }^{[7,8]}$ A recent study of residents of Hubei Province, reported that PTSD was experienced by 7\% of the total 285 individuals assessed for mental health. ${ }^{[9]}$ In Robbins et al. study $75.7 \%$ (81/107) of the radiology residents perceived either a moderate or marked decrease sense of morale and $61 \%(65 / 106)$ of program directors perceived either mild or marked decreased morale. ${ }^{[10]}$ Some of the main factors which negatively influenced the morale that were reported by radiology residents (RR) and program directors (PD) include the fear of loved ones contracting the disease (RR-75.7\% and PD- 76\%), fear of themselves contracting the disease (RR-78.5\% and PD-56\%), fear of being isolated/quarantined (RR-74.8\% and PD-52\%), concerns regarding lost block or elective opportunities (RR-63.6\%) and lack of guidance from department and institutional leadership accounted for $18.2 \%$ and $12.5 \%$, respectively for PD. ${ }^{[10]}$ This study highlights the need to adequately implement means to combat the short as well as long term psychological effects.

Apart from the above mentioned causes, the physical discomfort associated with the essential use of PPEs in cities with tropical climate such as Mumbai and Chennai has further added to the mounting burn-out amongst doctors and other health care workers. It is essential that a prompt and continuous channel of communication is established between the residents, other senior consultants and the mental health counsellors to address their concerns before the stage of burnout is reached. Residents wellness is closely associated with the clarity, adequacy, and reliability of the relevant information. While there is very little attention given to the educational activities, the head of the department and senior faculty should discuss with the residents regarding

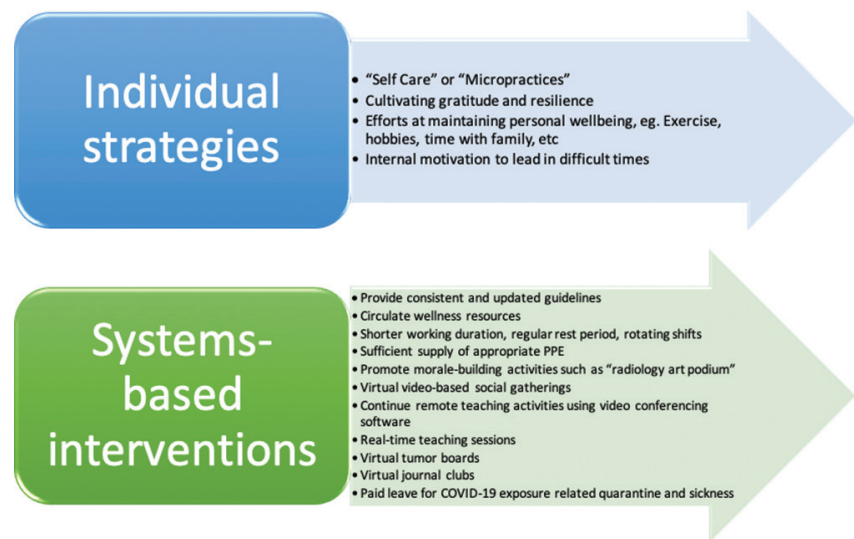

Figure 2: How to cope with the Psychological Impact of COVID-19 
the curriculum of the online/virtual teaching programmes and encourage maximum attendance of the teaching staff so that there is enough adequate interaction and flow of knowledge which will particularly help the exam going residents. Also the junior residents are finding it difficult to diagnose the various pathological conditions relating to COVID-19 without proper guidance and the shortage of senior staff in the department. To combat such situations, the senior staff should make sure that they are available to help the residents with their doubts and uncertainity of diagnosis via PACS and screen share applications. While the trainees are being designated non-clinical/clinical duties in various other departments such as diagnostic labs and ICU, discussions concerning recommendations on PPE usage, viral testing, self-quarantine, and return to work after illness should be encouraged in the departmental meetings that can instill self-confidence amongst these individuals. According to a study, the health care workers in a Beijing hospital who were either quarantined or worked in high-risk clinical settings such as SARS units or had family or friends who were infected with SARS, had substantially more post-traumatic stress symptoms than those without these experiences. They also reported depression, anxiety, fear, and frustration. ${ }^{[11]}$

Guidelines for financial as well as professional backup support in case of personal or family emergencies should be discussed to further alleviate the stress. In the over-burdened departments, the residents can possibly develop insomnia, phobias, anxiety, depression, panic, delirium, and even suicidal tendencies. Some health care professionals face isolation and negligence from their family members and neighbors as a reaction to fear of contracting disease. This can hamper their ability to deliver good health care to the patients and maintain peaceful decorum in the department.

The psychological problems faced by the senior consultants are the fear of pay-cuts and losing jobs accountable to the decreased elective workload. The head of the departments should acknowledge the additional demands that the pandemic is creating and advocate for resources to reinforce personal resilience (e.g., financial assistance for those with educations or other loans, adequate communication with the hospital management, accessible psychotherapy, online meditation/yoga/physical training sessions) for trainees as well as the consultant doctors. Considering insomnia is possibly the first casualty of such outbreak, it is therefore important to educate on sleep deprivation and insomnia and find ways to restore sleep and ensure enough rest. Most people cope very well in their own way and benefit a great deal of a relatively small quotient of shared concern, good information, and support.

When individuals are combating such a crisis, they should be made to feel well protected and supported, physically and emotionally. All the staff members should be made aware of "Self-care/micropractices". ${ }^{[12]}$ Self-care may include the following: individual routine with adequate breaks and sleep, healthy diet and exercise, maintaining regular contact with family and friends (Social media), engage in leisure activities and hobbies, relaxation exercises such as yoga. Families and the society should be made aware of the selfless contribution that the health care professional are making by means of expressing and spreading the sense of gratitude towards these individuals through online media and on social platforms. All efforts to overcome interpersonal isolation, from joke sharing in the nursing station to conference calls, can play significant role in times of severe physical strain and mental stress.In addition, health workers and patients should receive clear communication with regular and accurate updates regarding the 2019- nCoV outbreak, in order to address their sense of uncertainty and fear. Safe communication channels for patients and their families, such as smartphone communication compatible with applications such JIO MEET, FLOOR, SAY NAMASTE, KL MEET \& AIR MEET should be encouraged to decrease isolation. Teaching programs should continue through video lectures/webinars.

Every hospital should engage in formation of a multidisciplinary mental health care teams established by health authorities at regional levels (including psychiatrists, psychiatric nurses, clinical psychologists, and other mental health workers) which can deliver counselling sessions and emotional support to patients as well as the health care workers. Specialized psychiatric treatments and appropriate mental health care personnel should be designated for health care workers with pre-existing mental disorders. Such individuals can be seen by the counselors on a weekly-basis appointments scheduled in the department itself. Doctors with co-morbid health conditions such as diabetes, hypertension, pregnancy or with children should be given special consideration and consulted regarding allotting the postings and blocks to minimise physical/emotional breakdowns; also, childcare is not suffered. Mental health status updates should be given to their family and friends to further ensure emotional backup and less burn-out in terms of familial expectations and avoidable duties. For those residents who have been quarantined, secure services should be set up to provide psychological counseling using electronic devices and above-mentioned online meeting applications which also serve to decrease isolation amongst residents and the faculty working from home. For most patients and health workers, emotional, and behavioral responses are part of an adaptive response to extraordinary stress, and psychotherapy techniques such as those based on the stress-adaptation model might be helpful.

Psychological issues and mitigation plan relating to the radiographers

Radiographers form the building blocks of the radiology department and for a stronger foundation their physical, emotional, and financial needs and expectations should 
be timely recognized and given due consideration. For most radiographers, with very little knowledge of the PPE usage, adequate personal protection is becoming a major concern. ${ }^{[13,14]}$ It is not only having physical apprehensions but also psychological impact which is translating into stigmatic and seldom hostile attitude towards COVID-19 patients. With many not belonging to strong financial background are facing financial challenge way beyond imagination. They are either self-isolating or are facing unkind treatment from neighbours/landlords. At such instances it becomes prudent that the leaders and the other medical staff make them feel protected and supported. They should be educated regarding the proper use of PPE and the sanitization protocol. They should not be left alone in the departments and work stations such as the CT/MRI consoles to keep their confidence and morals boosted. Frequent exchange of greetings and light-hearted conversations help ease out stress. Head of the department/private consultants should have daily discussions with them, one on one and give them an opportunity to voice their concerns regarding any financial/emotional crisis and help them find a solution. While IRIA is coming up with distressing activities such as singing, painting and dancing competitions (Radiology ART PODIUM), we must involve the radiographers to perform with the radiologists to inculcate a sense of team effort in the department. ${ }^{[15]}$

To conclude, although the main focus of all health care individuals has constantly been to reduce the number of new cases by minimal transmission, save lives of the infected patients by findings effective cure and carry out meticulous research to develop preventive vaccine, we have been unknowingly giving very little consideration to critical issues relating to the psychological well-being of the front liners. It is of utmost necessity that the psychological impact of this pandemic needs to be recognized and dealt with, in a timely fashion. While planning interventions to fight the pandemic, mental health issues must not be sidelined instead considered pragmatically. The radiology departments must come up with a mitigation plan to combat the subsequent short-term and long-term adverse effects of COVID-19 on mental well-being of their staff members.

Financial support and sponsorship

Nil.

\section{Conflicts of interest}

There are no conflicts of interest.

\section{References}

1. Available from: https://edition.cnn.com/2020/05/17/opinions/covid19-worldwide-response-metzl/index.html. [Last accessed on 2020 July 10].

2. Fessell D, Cherniss C. Coronavirus disease 2019 (COVID-19) and beyond: Micropractices for burnout prevention and emotional wellness. J Am Coll Radiol 2020;17:746-8.

3. Fessell D, Garver K, Lexa F. Navigating "down time" during COVID-19. Acad Radiol 2020;27:862-3.

4. Restauri N, SheridanMD AD. Burnout and PTSD in the COVID-19 pandemic: Intersection, impact and interventions. J Am Coll Radiol 2020;17:921-6.

5. Massat MB. Radiology matters: Staying focused on radiologist wellness.

6. Patel RS, Bachu R, Adikey A, Malik M, Shah M. Factors related to physician burnout and its consequences: A review. Behav Sci (Basel) 2018;8:98.

7. Lee SM, Kang WS, Cho AR, Kim T, Park JK. Psychological impact of the 2015 MERS outbreak on hospital workers and quarantined hemodialysis patients. Compr Psychiatry 2018;87:123-7.

8. Phua DH, Tang HK, Tham KY. Coping responses of emergency physicians and nurses to the 2003 severe acute respiratory syndrome outbreak. Acad Emerg Med 2005;12:322-8.

9. Liu N, Zhang F, Wei C, Jia Y, Shang Z, Sun L, et al. Prevalence and predictors of PTSS during COVID-19 outbreak in China hardest-hit areas: Gender differences matter. Psychiatry Res 2020:112921.

10. Robbins JB, England E, Patel MD, DeBenedectis CM, Sarkany DS, Heitkamp DE, et al. COVID-19 Impact on well-being and education in radiology residencies: A survey of the association of program directors in radiology. Acad Radiol 2020;27:1162-72.

11. Wu P, Fang Y, Guan Z, Fan B, Kong J, Yao Z, et al. The psychological impact of the SARS epidemic on hospital employees in China: Exposure, risk perception, and altruistic acceptance of risk. Can J Psychiatry 2009;54:302-11.

12. Unadkat $S$, Farquhar M. Doctors' wellbeing: Self-care during the covid-19 pandemic. BMJ 2020;368:m1150. doi: 10.1136/ bmj.m1150.

13. Mohakud S, Ranjan A, Naik S, Deep N. COVID-19 preparedness for portable x-rays in an Indian hospital-Safety of the radiographers, the frontline warriors. Radiography 2020;26:270-7.

14. Hogg P, Holmes K, McNulty J, Newman D, Keene D, Beardmore C. Covid-19: Free resources to support radiographers. Radiography (Lond.) 2020;26:189-91.

15. Available from: https://www.facebook.com/ groups/2852900111499077/about/.[Last accessed on 2020 Jul 10]. 